\title{
Clinical evaluation of automated refraction in anterior chamber pseudophakia
}

\author{
Palaniswamy Sunder Raj, Jose R Villada, Kyaw Myint, Anne E Lewis, Tayo Akingbehin
}

\begin{abstract}
Automated refraction with the Canon RK-1 Autoref keratometer was evaluated in 85 eyes of 85 patients with an anterior chamber intraocular lens implant and a best corrected visual acuity of at least $6 / 12$. Autorefraction readings were obtained in $80(94 \%)$ of these eyes. The agreement between autorefraction and clinical refraction data was $89 \%$ for spherical equivalence $<0.51$ dioptres $(D), 91 \%$ for sphere power $<0.51 \mathrm{D}, 82 \%$ for cylinder power $<0.51 \mathrm{D}$, and $91 \%$ for cylinder axis $<11^{\circ}$. Autorefraction provides acceptably accurate postoperative refraction values in anterior chamber pseudophakia.
\end{abstract}

Autorefractors (ARs) are being increasingly used in busy ophthalmology clinics because they can be operated by a nurse or technician, ${ }^{1}$ are much quicker than manual refraction, ${ }^{12}$ and are highly esteemed by most patients. ${ }^{1}$ Objective autorefraction can provide reliable and valid preliminary refraction data, ${ }^{1-12}$ especially in subjects who do not possess 'true' accommodation due to aphakia, ${ }^{213}$ pseudophakia, ${ }^{14}$ or cycloplegia. ${ }^{121516}$

Intraocular lenses (IOLs) can interfere with the accuracy of objective ARs ${ }^{1910} 17$ by scattering the measuring infrared beam and increasing 'noise'. ${ }^{10}{ }^{17}$ Modern ARs overcome this problem by providing an 'IOL setting' which improves the signal-to-noise ratio in the recording system while refracting pseudophakes. We evaluated the use of one such instrument, the Canon RK-1 Autoref keratometer, in patients with posterior chamber IOLs and found it to be acceptably accurate. ${ }^{14}$ In this paper we report on the use of the same $A R$ in patients with anterior chamber IOLs.

\section{Patients and methods}

\section{INSTRUMENT}

The Canon RK-1 Autorefkeratometer combines both automated refraction and keratometry in one instrument. The optical principle on which it operates, its capacity to refract, special facilities possessed, and the method used to measure refraction with this instrument have been detailed in a previous communication. ${ }^{14}$

Ophthalmology, District General Hospital, Southport PR8 6NJ P Sunder Raj J R Villada K Myint A E Lewis T Akingbehin Correspondence to: Mr Tayo Akingbehin, FRCS. Accepted for publication 27 September 1990 PATIENTS AND EYES

This study was carried out on patients who had undergone anterior chamber IOL implantation in one or both eyes. Of the 102 such patients seen consecutively in the eye clinic 99 had unilateral anterior chamber IOLs and three were bilateral. For the purposes of this study we included only the right eyes of the patients with bilateral anterior chamber IOLs, giving us 102 eyes of 102 patients. Seventeen eyes were excluded because they did not attain a best corrected visual acuity of at least $6 / 12$. Of the remaining 85 eyes studied, valid $A R$ values could not be obtained owing to various causes in five eyes, leaving 80 eyes of 80 patients for analysis.

\section{METHODS}

All the refractions, clinical as well as on the autorefractor, were carried out without the use of mydriatics. Clinical refraction, streak retinoscopy followed by subjective verification, was carried out by two of the authors (PSR, KM) to achieve the best possible visual acuity. Autorefraction was performed to obtain a series of three readings by a different examiner (AEL). The 'standard' refractive value in the AR printout data (categorised into reliable and less reliable data) was then compared with the result of clinical refraction for degree of agreement.

\section{STATISTICAL ANALYSIS}

The statistical significance of the difference between the less reliable (indicated by the 'reliability mark') and reliable autorefractive data was evaluated using two tests: (1) $\chi^{2}$ test on the number of eyes in the two groups within $0.51 \mathrm{D}$ of spherical equivalence, spherical power, or cylindrical power and within $11^{\circ}$ in cylindrical axis as determined by clinical refraction; and (2) standard error of the difference between means on the mean difference in the various refractive components between automated and conventional refraction.

\section{Results}

Of the 85 eyes ( 85 patients) with anterior chamber IOLs which were eligible for the study valid autorefractive data could not be obtained in five eyes. The reasons included inability to keep the eyes focused on the target (two eyes), cylinder value outside the range of the AR, uncontrolled blinking, and inability to extend the neck (one eye each). Clinical refraction of the eye whose cylinder value could not be estimated by the AR revealed a cylinder power of $+5 \cdot 0 \mathrm{D}$.

The percentage agreement between autorefraction and clinical refractive data with respect to the various refractive components is values were within $0.51 \mathrm{D}$ of spherical equivalence, $91 \%$ were within $0.51 \mathrm{D}$ of spherical power and $11^{\circ}$ of cylindrical axis, and $82 \%$ were within $0.51 \mathrm{D}$ of cylinder power. The percentage agreement between the AR and clinical refractive data 
Table 1 Degree of agreement between autorefractive and clinical refractive data of various refractive components

\begin{tabular}{llll}
\hline & \multicolumn{3}{l}{ Autorefractor reading: no. (\%) } \\
\cline { 2 - 4 } Refractive component & $\begin{array}{l}\text { Reliable } \\
(n=57)\end{array}$ & $\begin{array}{c}\text { Less reliable } \\
(n=23)\end{array}$ & $\begin{array}{l}\text { Total } \\
(n=80)\end{array}$ \\
\hline Spherical equivalent & & & \\
$\pm 0 \cdot 25 \mathrm{D}$ & $35(61 \%)$ & $14(61 \%)$ & $49(61 \%)$ \\
$\pm 0.50 \mathrm{D}$ & $53(93 \%)$ & $18(78 \%)$ & $71(89 \%)$ \\
$\pm 1.00 \mathrm{D}$ & $56(98 \%)$ & $21(91 \%)$ & $77(97 \%)$ \\
Sphere power & & & \\
$\pm 0 \cdot 25 \mathrm{D}$ & $44(77 \%)$ & $14(61 \%)$ & $58(73 \%)$ \\
$\pm 0.50 \mathrm{D}$ & $56(98 \%)$ & $16(70 \%)$ & $72(91 \%)$ \\
$\pm 1 \cdot 00 \mathrm{D}$ & $57(100 \%)$ & $21(91 \%)$ & $78(99 \%)$ \\
Cylinder power & & & \\
$\pm 0.25 \mathrm{D}$ & $37(65 \%)$ & $8(35 \%)$ & $45(56 \%)$ \\
$\pm 0.50 \mathrm{D}$ & $48(84 \%)$ & $18(78 \%)$ & $66(82 \%)$ \\
$\pm 1.00 \mathrm{D}$ & $55(96 \%)$ & $20(87 \%)$ & $75(93 \%)$ \\
Cylinder axis & & & \\
$\pm 5^{\circ}$ & $43(75 \%)$ & $17(74 \%)$ & $60(75 \%)$ \\
$\pm 10^{\circ}$ & $53(93 \%)$ & $20(87 \%)$ & $73(91 \%)$ \\
$>10^{\circ}$ & $2(4 \%)$ & $2(9 \%)$ & $4(5 \%)$ \\
$\star$ NC & $2(4 \%)$ & $1(4 \%)$ & $3(4 \%)$ \\
\hline
\end{tabular}

$\mathrm{n}=$ Number of eyes (patients)

$\star$ No cylinder in one of the methods of refraction.

in the reliable and less reliable autorefractive data groups were compared by the $\chi^{2}$ test. There was no statistically significant difference between the two AR groups in spherical equivalence $(p>0.05)$, cylinder power $(p>0.5)$ or cylinder axis $(p>0 \cdot 3)$, but there was a significant difference in sphere power $(\mathrm{p}<0.005)$.

Autorefraction indicated a cylinder power in three eyes (one in the reliable group and two in the less reliable group) where none was found on clinical testing. The cylinder powers, in dioptres ('plus'), were $0.37,0.5$, and 0.75 respectively.

The mean differences between autorefraction and clinical refraction in spherical equivalence, sphere power, cylinder power, and cylinder axis are shown in Table 2. Spherical equivalence and the cylindrical power were both more myopic or less hypermetropic on the AR compared with conventional refraction unlike the sphere power, which was more hypermetropic or less myopic. The mean difference in cylinder axis was $4.2^{\circ}$ $\left(\mathrm{SD} 5 \cdot 2^{\circ}\right)$.

Autorefractive data were categorised into reliable and less reliable values. The differences between mean reliable AR values and clinical refraction data were computed and compared with values derived in a similar fashion for less reliable data. Using the standard error of the difference between the means to assess statistical significance, we found no difference between the two groups in spherical equivalence $(p>0.4)$, sphere power $(p>0.2)$, cylinder power $(p>0.4)$, and cylinder axis $(\mathrm{p}>0 \cdot 6)$.

Table 2 Mean difference and standard deviations (SD) between autorefraction and clinical refraction

\begin{tabular}{|c|c|c|c|}
\hline \multirow[b]{2}{*}{ Refractive component } & \multicolumn{3}{|c|}{ Autorefractor reading ${ }^{\star}:$ mean $(S D)$} \\
\hline & $\begin{array}{l}\text { Reliable } \\
(n=57)\end{array}$ & $\begin{array}{l}\text { Less reliable } \\
(n=23)\end{array}$ & $\begin{array}{l}\text { Total } \\
(n=80)\end{array}$ \\
\hline $\begin{array}{l}\text { Spherical equivalent } \\
\text { (D) } \\
\text { Sphere power (D) } \\
\text { Cylinder power (D) } \\
\text { Cylinder axis (degrees) }\end{array}$ & $\begin{aligned}-0.05(0.27) \\
0.00(0.22) \\
-0.04(0.44) \\
4 \cdot 1(5 \cdot 7)\end{aligned}$ & $\begin{array}{l}0.05(0.68) \\
0.14(0.55) \\
-0.14(0.55) \\
4.6(5.0)\end{array}$ & $\begin{array}{r}-0.01(0.46) \\
0.04(0.35) \\
-0.02(0.48) \\
4.2(5.2)\end{array}$ \\
\hline
\end{tabular}

\section{Discussion}

Extracapsular cataract extraction followed by posterior chamber IOL implantation is the current method of choice in the visual rehabilitation of cataract patients. Anterior chamber IOL implantation is still indicated as a primary procedure where posterior chamber IOL implantation is not possible owing to extensive rupture of the posterior capsule or zonular dehiscence. Secondary implantation in aphakia is usually performed using an anterior chamber IOL. With preoperative ocular biometry the desired postoperative refraction can be achieved by the surgeon in a significant number of pseudophakic eyes. ${ }^{18}$ However, ocular refraction will continue to play an important role in the postoperative evaluation of pseudophakic eyes not only for optimising visual acuity but also for managing postoperative astigmatism.

An autorefractometer which can be operated by the nursing staff to provide accurate refraction values in pseudophakes will result in an appreciable saving of the clinician's time. Previous reports on autorefraction in pseudophakic eyes have not been adequately comprehensive ${ }^{1-3}$ or have dealt exclusively with posterior chamber pseudophakes. ${ }^{14}$ Rassow and Wesemann ${ }^{1}$ classified results as good, bad, or instrument failure in terms of the visual acuity achieved with the autorefractor reading compared with subjective refraction. Wood ${ }^{10}$ found that IOLs accounted for a significant number of unobtainable or rejected AR measurements. Dufier et al ${ }^{2}$ studied a general clinic population which included normal, aphakic and pseudophakic eyes but pseudophakes were not separately analysed.

In this study of anterior chamber IOLs the various autorefractive data in pseudophakic eyes were all acceptably accurate especially as preliminary refraction. Subjective verification of the result was essential before final prescription. A similar outcome with the AR has been reported in posterior chamber pseudophakes ${ }^{14}$ and adults and children under cycloplegia. ${ }^{15} 16$ In comparison manifest autorefraction in normal subjects has yielded poorer results for spherical equivalence with percentage agreement with clinical refraction being as low as $41 \% .^{12}$ This is probably because the inbuilt autofogging mechanism in the AR fails to neutralise adequately the patients' accommodation, producing significant instrument myopia. ${ }^{15} 16$ This phenomenon does not affect aphakes or pseudophakes who lack 'true' accommodation, ${ }^{19}$ accounting for autorefraction results which are superior to those observed in subjects with intact accommodation. ${ }^{12410} 121415$

Although differential analysis of the reliable and less reliable AR reading showed a statistically significant difference between the two groups in sphere power, this was not clinically important, ${ }^{14}{ }^{15}$ as the autorefractive data were considered as preliminary results needing subjective verification.

It is not only the accuracy of the autorefraction measurements that is important but also the percentage of a given number of eyes that can be measured with an AR. AR readings were either invalid or not obtainable in $6 \%$ (five out of 85 ) of the eyes studied where conventional refraction was possible. This is lower than the $25 \%{ }^{10}$ and 
$60 \%{ }^{4}$ mentioned in previous studies on autorefraction, which have included pseudophakic eyes, but is in agreement with our study of posterior chamber IOLs. ${ }^{14}$

A single eye could not be measured by the AR because the cylinder value was supposedly outside its specified range. On clinical refraction the cylinder power in this eye was $+5 \mathrm{D}$. The cylindrical power determined clinically was less than that indicated objectively by autorefraction, possibly because the patient with this eye had difficulty in accepting subjectively the high cylindrical correction. Three of the study eyes which had a cylinder power on the AR did not need it on subjective verification. Previous reports ${ }^{74}$ have pointed out that cylinder values obtained on the AR were often clinically insignificant.

The clinical impression gained during this evaluation was that autorefraction was both quick and easy to perform. Although this procedure was not timed and compared with conventional refraction, a saving in the clinician's time of four minutes per eye has been reported in a clinic setting. ${ }^{2}$ The results of this evaluation of the Canon RK-1 Autoref keratometer in refracting anterior chamber pseudophakes were consistent with those of our previous study on posterior chamber pseudophakes. ${ }^{14}$

We thank Miss Lisa Jones for secretarial assistance.
1 Rassow B, Wesemann $W$. Automatic infra-red refractors. Ophthalmology 1984; 91(S): 10-26.

2 Dufier JL, Abitbol M, Pigamo F, Prete T, Paris JP, Poitreuand $O$. Automatic objective refractometer: evaluation in 3618 eyes. F Fr Ophtalmol 1987; 10: 301-8.

3 Guyton DL. Automated refractors - 1983. Ophthalmology $1983 ; 90(9 S): 36-44$

4 French CN, Wood ICJ. The dioptron in practice. Optician 1981; 181: 18-30.

5 Wong EK, Patella V, Pratt M, Myers S, Gaster R, Irving HL. Clinical evaluation of the Humphrey automatic refractor. Arch Ophthalmol 1984; 102: 870-5.

6 Wood ICI, Papas E, Burghardt D, Hardwick G. A clinical evaluation of the Nidek autorefractor. Ophthalmic Physiol Opt 1984; 4: 169-78.

7 Ghose S, Nayak BK, Singh JP. Critical evaluation of the NR1000F autorefractometer. Br $\mathcal{F}$ Ophthalmol 1986; 70: 221-6.

8 Reddy NS, Agarwal S. Clinical evaluation of the Topcon autorefractor. Indian $\mathcal{f}$ Ophthalmol 1987; 35: 407-10.

9 Wesemann W, Rassow B. Automatic infra-red refractors - a comparative study. Am $\mathcal{F}$ Optom Physiol Opt 1987; 64: 62738.

10 Wood ICJ. A review of autorefractors. Eye 1987; 1: 529-35.

11 Anonymous. Refractors. Ophthalmology 1988; 95(S): 171-6.

12 Ehrlich DL. The Canon RK-1: refractor mode evaluation. Ophthalmic Physiol Opt 1990; 10: 97.

13 Guillon M. Automated refraction in aphakia-II. Its repeatability and accuracy compared to conventional techniques. ability and accuracy compared to con

14 Sunder Raj P, Akingbehin T, Levy AM. Objective autorefraction in posterior chamber pseudophakia. Brf Ophthalmol (in press).

15 Nayak BK, Ghose S, Singh JP. A comparison of cycloplegic and manifest refractions on the NR-1000F (an objective autorefractometer). Br $\mathcal{O}$ Ophthalmol 1987; 71: 73-5.

16 Hunold W, Auffarth G, Effert R. Clinical application of the Canon R-10 autorefractor in children with squint. Klin Monatsbl Augenheilkd 1988; 192: 58-65.

17 Charman WN. Infra-red autorefractors - here to stay? Ophthalmic Physiol Opt 1985; 5: 237-9.

18 Dang MS, Sunder Raj PP. SRK II formula in the calculation of intraocular lens power. Br f Ophthalmol 1989; 73: 823-6.

19 Nakazawa $M$, Ohtsuki K. Apparent accommodation in pseudophakic eyes after implantation of posterior chamber intraocular lenses. Am $\mathcal{F}$ Ophthalmol 1983; 96: 435-58. 